\title{
A Position-Based Clustering Technique for Ad Hoc Intervehicle Communication
}

\author{
Zhigang Wang, Member, IEEE, Lichuan Liu, Member, IEEE, MengChu Zhou, Fellow, IEEE, \\ and Nirwan Ansari, Senior Member, IEEE
}

\begin{abstract}
Intervehicle communication is a key technique of intelligent transport systems. Recently, ad hoc networking in the vehicular environment was investigated intensively. This paper proposes a new clustering technique for large multihop vehicular ad hoc networks. The cluster structure is determined by the geographic position of nodes and the priorities associated with the vehicle traffic information. Each cluster elects one node as its cluster head. The cluster size is controlled by a predefined maximum distance between a cluster head and its members. Clusters are independently controlled and dynamically reconfigured as nodes move. This paper presents the stability of the proposed cluster structure, and communication overhead for maintaining the structure and connectivity in an application context. The simulation is performed with comparative studies using CORSIM and NS-2 simulators.
\end{abstract}

Index Terms-Ad hoc wireless network, cluster, intelligent transportation, sensor network, vehicular network.

\section{INTRODUCTION}

I NTERVEHICLE communication is one of the four key systems defined in intelligent transportation system architecture [23]. The intervehicle communication enables each driver or each vehicle to communicate with other vehicles at any location at any time. Vehicles equipped with wireless communication devices are no longer isolated systems, and can instantly form vehicular ad hoc networks (VANETs) [9], [13], [15], [16], [18], [21]. Two passing vehicles can exchange data (single hop), or data can pass several other vehicles when they act as routers/relays (multihop) [20]. With this principle, highly efficient accident warning systems are possible; cars involved in an accident can send warning messages back over a predefined number of other vehicles, thus avoiding motorway pileups and enhancing the traffic safety. VANETs extend the horizon of drivers and automated systems in that the intervehicle communi-

Manuscript received July 26, 2005; revised May 22, 2006 and September 1, 2007. This work was supported by the Chang Jiang Scholars Program, Ministry of Education, China. This paper was recommended by Associate Editor S. X. Yang.

Z. Wang is with the Center for Advanced Vehicular Systems, Mississippi State University, Starkville, MS 39759 USA (e-mail: zw62@msstate.edu).

L. Liu is with the Department of Electrical Engineering, Northern Illinois University, Dekalb, IL 60115 USA (e-mail: lichuan@ceet.niu.edu).

M. C. Zhou is with the Department of Electrical and Computer Engineering, New Jersey Institute of Technology, Newark, NJ 07102 USA, and also with the School of Electro-Mechanical Engineering, Xidian University, Xían 710071, China (e-mail: zhou@njit.edu).

N. Ansari is with the Department of Electrical and Computer Engineering, New Jersey Institute of Technology, Newark, NJ 07102 USA (e-mail: ansari@njit.edu).

Color versions of one or more of the figures in this paper are available online at http://ieeexplore.ieee.org.

Digital Object Identifier 10.1109/TSMCC.2007.913917 cation not only helps the driver acquire real-time traffic information and react quickly, but also facilitates smooth driving according to varying transportation conditions. For example, drivers can change their route plan in case of traffic pileup, and also their vehicles can adjust the fuel economy, brake systems, or airconditioning status based on the current traffic condition. Therefore, the safety and efficiency of the road traffic are improved.

However, VANET is fundamentally different from a typical mobile ad hoc network (MANET) with respect to the dominating models in MANET research [3], [5], [22]. Driver behavior, constraints on mobility, and high speeds contribute unique characteristics in VANET. In particular, [3] quantifies these differences as rapid topology changes, frequent fragmentation, small effective network diameters, and limited redundancy. Due to the relative speeds of vehicles, the topology of VANET keeps rapidly changing even though the movement of vehicles is somewhat predictable, i.e., they must stay on the roadway and have the same moving direction. A message path can typically survive 1 min while maintaining a transmission range of 500 $m$ [9], [18]. Consequently, a VANET may suffer frequent partition, and hence, costly overheads for exchanging new topology information and reconfiguring each node [19]. Aoki and Fuji [3] also show that a typical effective network diameter is no larger than 9 hops. Moreover, limited temporal redundancy of roadways discourages the application of some routing protocols, which require multiple paths.

Traditional topology management approaches are not designed for the earlier vehicular environment. For example, energy efficiency for regular MANET is a critical issue but not for VANET, since vehicles are capable of recharging battery during their journey. More importantly, vehicle mobility related to transportation information should be considered together with communication issues. Based on the previous observation, we propose position-based prioritized clustering (PPC), a new topology control method for VANET. This proposal incorporates position information into a novel hierarchical clustering technique. In order to justify this proposal, a microscopic vehicle traffic simulator, CORSIM, is deployed along with NS-2, a widely used computer network simulator, to derive the results and conclusions.

The rest of the paper is organized as follows. Section II describes the PPC approach. Section III analyzes the stability of cluster and the needed communication overhead using a probabilistic model. Section IV shows the performance using simulations. Section V summarizes the paper and its key contributions to enable intelligent transportation. 
$\left(3,5,9_{3}, 6,0.42\right)$

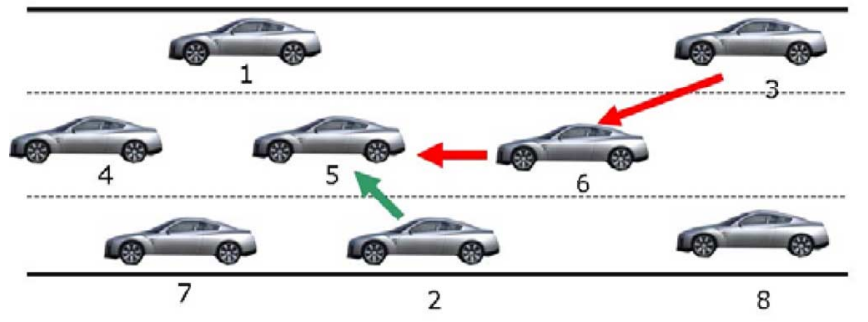

$\left(2,5, g_{2}, 2,0.35\right)$

Fig. 1. Example of clustering vehicles disseminating cluster information.

\section{Position-Based Prioritized Clustering TechniQue}

\section{A. Overview and Assumption}

Clustering is a technique to group nodes in a network into logically separated entities called clusters. Clustering can simplify such essential functions as routing, bandwidth allocation, and channel access. Several heuristic clustering techniques have been proposed to choose cluster heads in an ad hoc network. These are lowest ID, highest degree, and node-weight heuristics [1], [5], [7]. However, they cannot be deployed directly in ad hoc vehicular networks, since their design objectives are not for high-mobility vehicular networks. For example, vehicles leave and enter highways randomly and rapidly, the computation task, then, causes a huge amount of communication overheads for each node to perform the heuristic technique based on the highest-degree algorithm. Cluster heads may frequently change their relative position on highways, and then, the size and stability of clusters change unpredictably if lowest ID and node-weight heuristics are used. On the other hand, vehicles on (one way) highways have almost the same direction within a certain area. Therefore, their geographical location and velocity information are helpful when they are evenly divided into nonoverlapping clusters along highways. [14] demonstrated that better performance could be achieved if the geographic position of the network nodes is known.

This work assumes that each node is assigned a distinct ID, which is greater than zero. For the clustering purpose, each node maintains a small amount of information of itself and its neighboring nodes. Periodically, a node broadcasts the information, which is referred to as its cluster information denoted by a 5 -tuple $\left(i, h, g, i_{N}, p\right)$, where

$i: \quad$ node ID;

$h$ : cluster head ID;

$g$ : node geographic location;

$i_{N}$ : ID of the next node along the path from the node to its cluster head;

$p$ : node priority.

Fig. 1 is an example network of clustering vehicles. Node 5 is the cluster head node and node 3 distributes its cluster message to its neighboring nodes. The meaning of cluster information is defined earlier; for example, $\left(3,5, g_{3}, 6,0.42\right)$ means that node 3 is using node 5 as its cluster head, and its geographic location is $g_{3}$, which is obtained from a GPS device, its next hop to the cluster head is node 6 , and its priority is 0.42 .

\section{B. Cluster Size and Minimal Dominating Set}

The major objective of clustering is to achieve relatively stable cluster structure, because frequent cluster reconfiguration generates tremendous communication load, which significantly reduces available bandwidth for message dissemination. Effective cluster size is both related to radio transmission range and vehicle traffic density. Therefore, cluster size may limit radio efficiency and throughput. The density is time varying [4], [17]. Hence, the cluster radius $L$ is defined in this paper, in order to organize the nodes into a cluster within the given maximum distance. When a node moves relatively away from its cluster head, and the distance between the node and its cluster head is larger than the cluster radius $L$, it joins a new cluster if it can find an existing cluster head within $L$; otherwise, a new cluster is formed with the node as the cluster head. When the distance between two cluster heads is detected to be less than or equal to a predetermined threshold, $D(D \leq L)$, the cluster with less members is dismissed. Each of the nodes in the dismissed cluster finds a new cluster to join. This paper will investigate the impact of transmission range and traffic parameter on $L$ and $D$.

The topology of VANET is represented by an undirected graph $G=(V, E)$, where $V$ is a set of network nodes and $E \subseteq V \times V$ is a set of links between nodes. The minimum dominating set problem in graph theory and the relevant minimum connected dominating set problem well describe the clustering approach to topology control [6]. Each node is either in a dominating set, or adjacent to a node in a dominating set. However, the problem of computing a minimum dominating set is known to be NP-hard [2], even when the complete network topology is known. Therefore, suboptimal solutions using a minimal dominating set (MDS), which is a subgraph of minimum dominating set and based on local minimum election of the dominators, should be used to approximate the calculation of the minimum dominating set. This work proposes a novel heuristic clustering approach to the election of cluster heads, which is equivalent to the computation of an MDS. The idea behind the approach is to make cluster heads form a quite stable backbone in highly dynamic vehicular environment. Therefore, partitioned connectivity caused by fragmentation is compensated to a certain degree.

\section{Cluster Head Election Algorithm}

We have three observations while designing the approach of cluster head election. First, most message packets forwarded in VANET travel locally, same as what occurs in regular ad hoc networks [8], [12]. A vehicle moving in a highway, for example, has little interest on distributing traffic pileup warnings to vehicles 100 miles away. Second, according to the definition of MDS, the cluster head election information for any node should include only nodes that are one hop or two hops away from the node itself, since every node is one hop away from a cluster head. Third, too many cluster heads around the same set of nodes lead to no MDS [1], [10], [11]. 


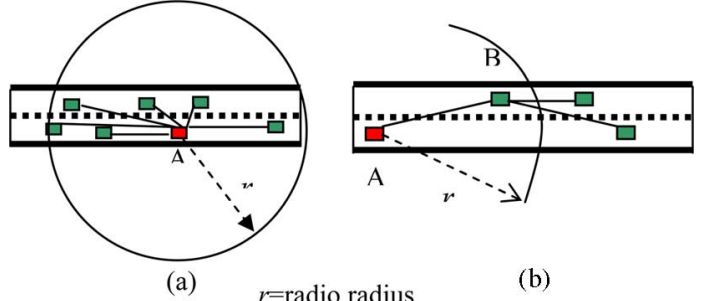

Fig. 2. Two conditions that make node A a cluster head.

Based on the earlier observations, every vehicle exchanges and maintains its neighbors' a priority value for each node information. The information is used for every node to calculate its priority in order to elect a cluster head among its two-hop neighborhood, such that any two nodes cannot have the same priority at the same instant during this stage. A node becomes a cluster head only if it satisfies the following two conditions.

1) The node has the highest priority in its one-hop neighborhood.

2) The node has the highest priority in the one-hop neighborhood of one of its one-hop neighbors.

Fig. 2 illustrates the two conditions that make node A the cluster head. Node A has the highest priority among its one-hop neighbors in Fig. 2(a). It has the highest priority among node B's one-hop neighbors in Fig. 2(b). The algorithm produces priorities distributing, which is normalized to $[0,1)$.

While designing the criteria of computing priorities, we make two reasonable assumptions. First, every vehicle has limited travel time and distance on highway. Second, two neighboring vehicles do not have the exact same instant speed along their journey. Note that they can certainly have the same average speed over an entire journey. In other words, the relative speed is not zero all the time. To take into account the mobility associated with transportation information and the timestamp during the election of membership of MDS, we define the following criteria.

1) To increase the stability of cluster heads in MDS, vehicles having a longer trip are more qualified for being elected as cluster heads. A vehicle, which would travel longer time, is assigned higher priority; hence, at the very beginning of starting its travel, the expected travel time of a vehicle is calculated and announced using its desired driving speed and the geographic information system once its driver sets the destination.

2) To avoid elected cluster heads losing connectivity with their neighbors very soon, the eligibility of a vehicle should decrease quickly when its velocity has big difference from the average speed. Thus, a vehicle with large speed deviation is assigned lower priority.

3) Once a cluster head is elected and a cluster is formed, recalculating priorities is necessary only if the cluster is dismissed, and therefore, each node should compute its new priorities following the previous rules.

Many possible solutions can be used to compute the priority of a node while considering the aforementioned criteria. We define

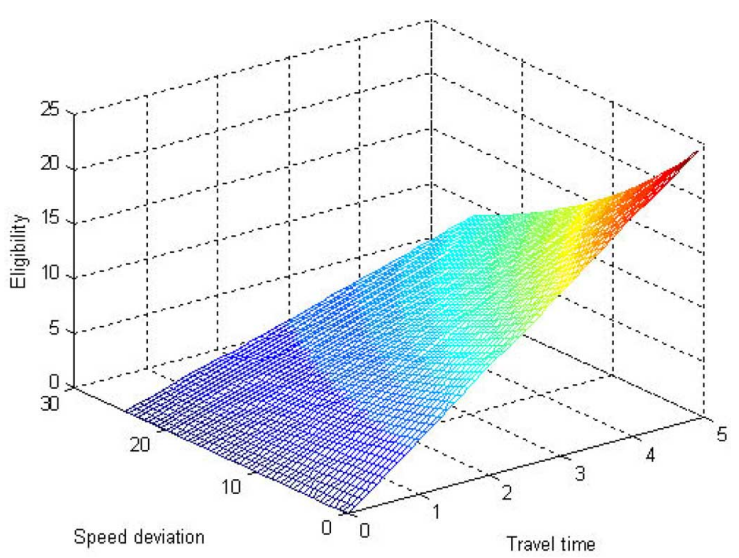

Fig. 3. Illustration of the eligibility function.

that the priority of node $i$ is given by the following formula:

$$
p_{i}=\operatorname{Hash}(t \oplus i) \oplus E_{i} .
$$

A hash function is used to generate a unique priority for node $i$ according to the input of node ID, current time, and the eligibility function. The eligibility function $E_{i}$ is defined by

$$
E_{i}=T e^{-0.2 d}
$$

where $T \in\left(0, T_{\max }\right)$ is the estimated travel time and $d \in[0,50]$ is the speed deviation. $T_{\max }$ is the maximal travel time. The units of $T$ and $d$ are minute and miles/hour, respectively. Fig. 3 illustrates the impact of the rules on the eligibility of a node. If a vehicle has long travel time and small speed deviation, its eligibility is high accordingly. To avoid the same eligibility value, each node must use a hash function to generate a unique normalized priority, as stated in (1).

\section{Cluster Registration and Updates}

When node $i$ is powered up, it sets its cluster information to be $\left(i, 0,0, g, p_{i}\right)$ indicating that it is at its registration phase. It, then, searches for its neighboring nodes. If there is, at least, one node, it sends its cluster information to all of them. Upon receiving the cluster information of node $i$, each neighboring node adds $i$ as one of its neighbors. Each neighboring node that detects the existence of $i$ also sends its cluster information to node $i$.

When $i$ receives the cluster information from all of its neighboring nodes, it checks whether there is any registered neighboring nodes that are not in the registration phase. If yes, it tries to find a cluster such that the distance between the cluster head and itself is minimum and is less than or equal to the cluster radius $L$. If such a cluster is found, it joins the cluster and updates its cluster information. It, then, sends its clustering information to all of its neighboring nodes. Upon receiving the cluster information of $i$, each neighboring node updates its maintained information.

If $i$ finds that: 1) it has no neighboring node; 2) none of its neighboring nodes belongs to a specific cluster; or 3) it cannot find a cluster head that is within the cluster radius $L$, it forms a new cluster with itself as the cluster head and sets its 


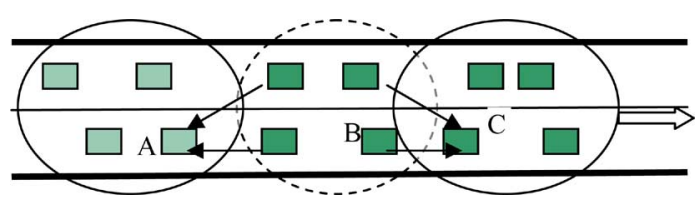

Fig. 4. Illustration of reconfiguration.

cluster information to be $\left(i, i, 0, g, p_{i}\right)$. Node $i$, then, sends its clustering information to all of its neighboring nodes, if any.

When $i$ detects the existence of a new neighbor, node $j$, and finds that $j$ is at its registration phase, $i$ replays its cluster information to $j$. Meanwhile, $i$ adds $j$ as a new neighbor and updates its information if $j$ decides to join this cluster. If $j$ is not at its registration phase and both $i$ and $j$ belong to the same cluster, node $i$ updates its cluster information and sends its new cluster information to all of its neighboring nodes. Otherwise, nothing else needs to be done.

When node $i$ receives the new cluster information from an existing neighbor node $j$, it updates the cluster information of $j$ in its routing table. If $j$ is the next node on the path from node $i$ to the cluster head, $i$ checks whether the new cluster information of $j$ is the same as the original one.

\section{E. Cluster Reconfiguration}

If the distance between two cluster head nodes is detected less than the dismiss threshold $D$, the cluster with fewer members is dismissed to reduce communication overheads while its members join other clusters. Each node of this cluster launches a new registration stage to join other clusters. The threshold determines the rate of cluster reconfiguration, and also, depends on the radio transmission range. It makes no sense when the distance between two cluster heads is less than the cluster diameter $2 L$. An example of reconfiguration is illustrated in Fig. 4.

\section{Performance AnAlysis of Stability}

\section{A. Model}

Assume that the spatial position of vehicles in a highway is confined to 1-D (line). But, in general, a two-lane highway network has different performance from that of a one-way four-lane highway network because of the influence of traffic capacity. Therefore, we model a cluster with a rectangle whose length is $2 L$, twice the cluster radius. First, we define the following terms:

$v: \quad$ average velocity of vehicles;

$\rho: \quad$ average vehicle density;

$n$ : number of lanes;

$w$ : width of roadway;

$r$ : efficient radio range;

$\Delta t$ : time headway (time between two following vehicles passing a reference point);

$K$ : $\quad$ average number of nodes in a cluster;

$d_{e}$ : effective distance between two vehicles.

Traffic patterns depend on the traffic density, i.e., the number of vehicles per kilometer. At high densities, the complex interactions between neighboring vehicles make the modeling of

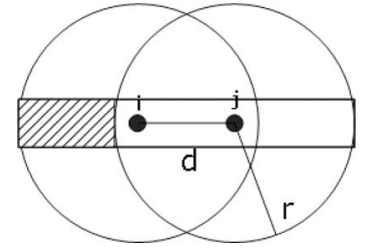

Fig. 5. Effective area when node $i$ becomes the cluster head.

such a dynamical system a challenge. Several vehicle spacing models are depicted in [19]. Here, an exponential distribution model is used with mean $N$.

\section{B. Cluster Stability}

Although this proposal uses a node-prioritized MDS election algorithm based on the transportation parameters, the performance of the algorithm can be evaluated regardless of node priorities. Therefore, we consider the case in which each node has equal priority to analyze the probability of a node being elected as a cluster head. The probability of a node winning over $k$ other contenders is $1 /(k+1)$. According to the previous model, the probability of a node winning among all contenders is

$$
\sum_{k=1}^{\infty} \frac{1}{k+1} \frac{N^{k}}{k !} e^{-N}=\frac{e^{N}-1-N}{N e^{N}} .
$$

The probability that a node has, at least, one contender is simply $1-e^{-N}$. If $N_{1}$ is the average number of one-hop neighbors of a node, for the first condition defined before, we have

$$
p_{1}=\sum_{k=0}^{\infty} \frac{N_{1}^{k}}{k !} e^{-N_{1}} \frac{1}{k+1} .
$$

There are too many situations that lead to the second condition. Hence, we only consider the lower bound of the probability, that node $i$ becomes a cluster head when considering its one-hop neighbor $j$ 's all neighboring nodes. Fig. 5 shows the relationship between nodes $i$ and $j$.

Since the roadway width is far less than the transmission range, only the nodes in the shaded area is effective for calculating the priority. So, the shaded area is approximately $w r$. According to the second condition, node $i$ should have a lower priority than the nodes in the shaded area, and, at the same time, have highest priority in node $j$ 's one-hop neighbors. Therefore, the probability of node $i$ having a lower priority than the nodes in the shaded area is

$$
p_{2}=\sum_{k=1}^{\infty} \frac{(w r)^{k}}{k !} e^{-w r} \frac{k}{k+1} .
$$

The probability that node $i$ has the highest priority among node $j$ 's one-hop neighborhood is

$$
p_{3}=\sum_{k=0}^{\infty} \frac{N_{1}^{k}}{k !} e^{-N_{1}} \frac{1}{k+2} .
$$

Since earlier events must happen simultaneously, the probability that node $i$ meets the second condition is $p_{2} p_{3}$. Apparently, 
the two conditions defined are mutually exclusive; hence, the probability of node $i$ becoming a cluster head is

$$
P_{\mathrm{ch}}=p_{1}+\left(1-p_{1}\right) p_{2} p_{3} .
$$

\section{Cluster Radius}

Because

$$
d_{e}=\frac{v \Delta t}{n}
$$

and

$$
K=\frac{2 L n}{d_{e}}
$$

hence

$$
\begin{aligned}
K & =\frac{2 L n^{2}}{v \Delta t} \\
L & =\frac{K v \Delta t}{2 n^{2}} .
\end{aligned}
$$

Because $\Delta t$ is a random variable, so is $K$. In [15], the distribution of time headway on highways is given. The typical mean time headway is $2-4 \mathrm{~s}$. From (4), we learn that the cluster radius is determined by the average velocity of vehicles, the number of nodes in a cluster, and the number of lanes. If the average velocity increases, the space between two vehicles must also increase to ensure driving safety. Then, a larger cluster radius is needed. On the other hand, when the number of lanes is doubled, the cluster radius needs to be reduced four times. It is also easy to understand the relationship intuitively. More lanes lead to larger traffic capacity, and more members of a cluster if the vehicle density is fixed. To guarantee certain level of available radio bandwidth, the cluster radius has to be reduced so that effective spatial and frequency reuse can be achieved.

\section{Dismiss Threshold}

In this proposal, a cluster is dismissed only when its cluster head is dismissed, and therefore, other nodes are reconfigured. When the distance between two cluster head nodes is less than or equal to the dismiss threshold $D$, clusters are reconfigured. Hence, we have the probability of cluster reconfiguration

$$
P(d<D)=\int_{0}^{D} \lambda e^{-\lambda x} d x=1-e^{-\lambda D} .
$$

The mean value of the given exponential distribution is $1 / \lambda$. Therefore, from the definition of the effective distance, we have

$$
\lambda=\frac{n}{v \Delta t} .
$$

From (12) and (13), we get

$$
P(\text { cluster_reformation })=1-e^{-n D / v \Delta t} .
$$

At last, we have

$$
P(\text { cluster_reformation })=1-e^{-K D / 2 L n} .
$$

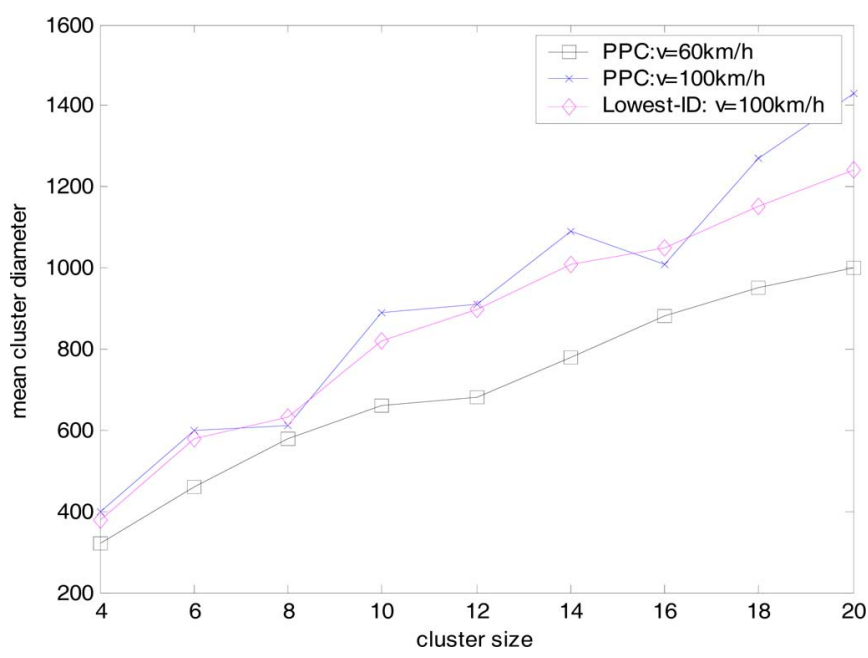

Fig. 6. Mean cluster diameter (two lanes) where PPC stands for position-based prioritized clustering.

According to (15), one can expect that a larger dismiss threshold leads to a higher rate of cluster head changes and higher probability of cluster reconfiguration. On the contrary, if $L$ increases, the probability decreases. Since the dismiss threshold is related to the transmission range, then, the probability of cluster changes is also related to the transmission range. Larger transmission provides longer distance for cluster heads to detect each other, and therefore, more frequent cluster reconfigurations occur. The next section shows the simulation results to demonstrate this conclusion.

\section{Simulation Results}

Extensive simulations are performed to study the characteristics of the proposed clustering technique using the microscopic vehicle traffic simulator, CORSIM, and the computer network simulator, NS-2. Developed by the Federal Highway Authority, CORSIM is able to provide vehicle traffic simulation data that are very close to real traffic data. The network used in the simulations consists of 100 vehicles moving in a highway with $10 \mathrm{~km}$ length. The packet length including the position information is 75 bytes. The traffic density is 50 vehicles $/ \mathrm{km}$ per lane. The time headway is $2 \mathrm{~s}$ and the effective radio transmission range is $250 \mathrm{~m}$. A comparative study of other clustering techniques is also conducted to evaluate the proposed method. Lowest ID and highest degree clustering methods are examined using the same configurations.

We first compare the mean cluster radius with different average cluster size when the average velocity changes. Fig. 6 shows that different average velocities affect the cluster diameter as discussed previously.

Then, simulations, as shown in Fig. 7, indicate that the number of lanes has significant influence on the cluster diameter due to higher traffic capacity.

One can also conclude that the mean cluster diameter is not strictly linear with cluster size, as (11) indicates. The main reason is that the movements of vehicles are not independent in the simulation experiments. 


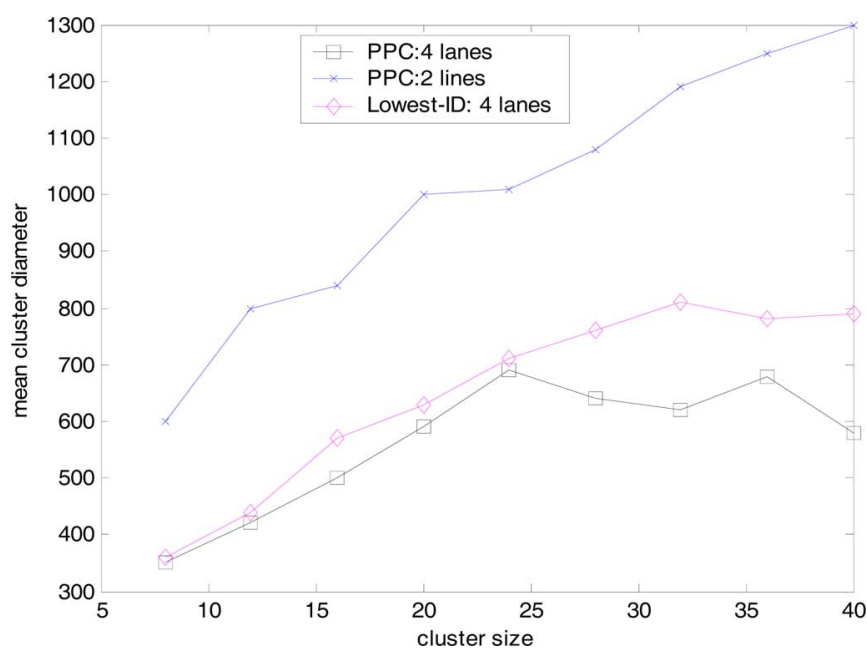

Fig. 7. Mean cluster diameter $(70 \mathrm{~km} / \mathrm{h})$.

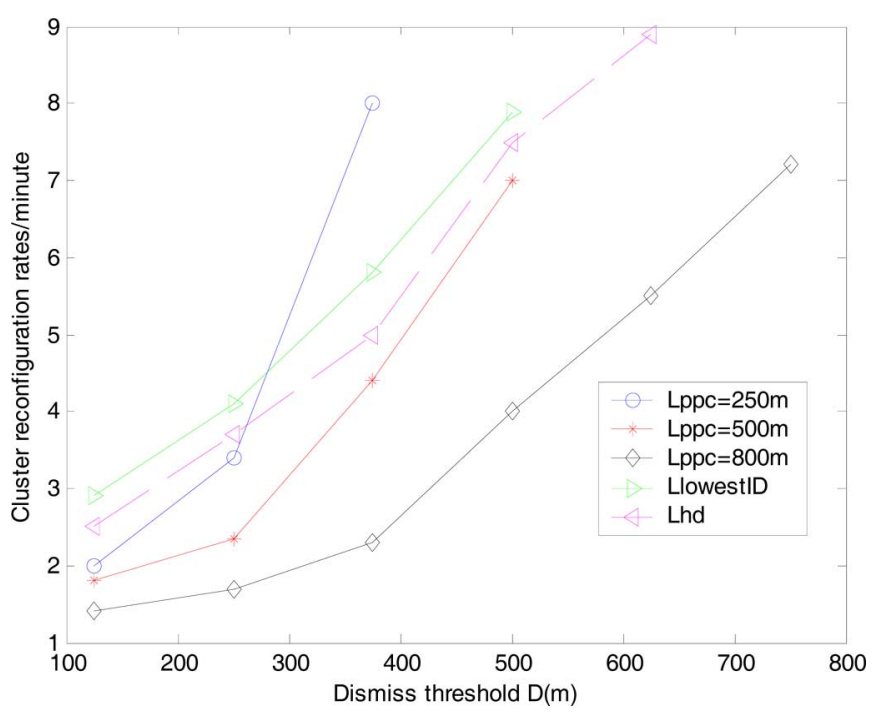

Fig. 8. Cluster reconfiguration rate versus dismiss threshold.

A good clustering algorithm should be stable to radio motion, i.e, it should not change the cluster configuration too drastically when a few nodes are moving and the topology changes rapidly. The dismiss threshold $D$ determines whether a cluster should be dismissed or not, and it is critical for cluster reconfigured rate. It is desirable that a clustering technique incurs low cluster reconfiguration rate. Figs. 8 and 9 show the cluster reconfiguration rates of the proposed technique for various values of cluster radius $L$ and cluster dismiss threshold $D$. The performance of the regular lowest ID and the highest degree techniques is also presented in Figs. 8 and 9, and their $L$ and $D$ are both fixed at $500 \mathrm{~m}$.

From the previous figures, the following observations are made: for a fixed value of the cluster radius $L$, the cluster reconfiguration rate increases as the cluster dismiss threshold $D$ increases. There are two reasons for this phenomenon. First, increasing the cluster dismiss distance increases the probability that the distance between two cluster heads becomes less

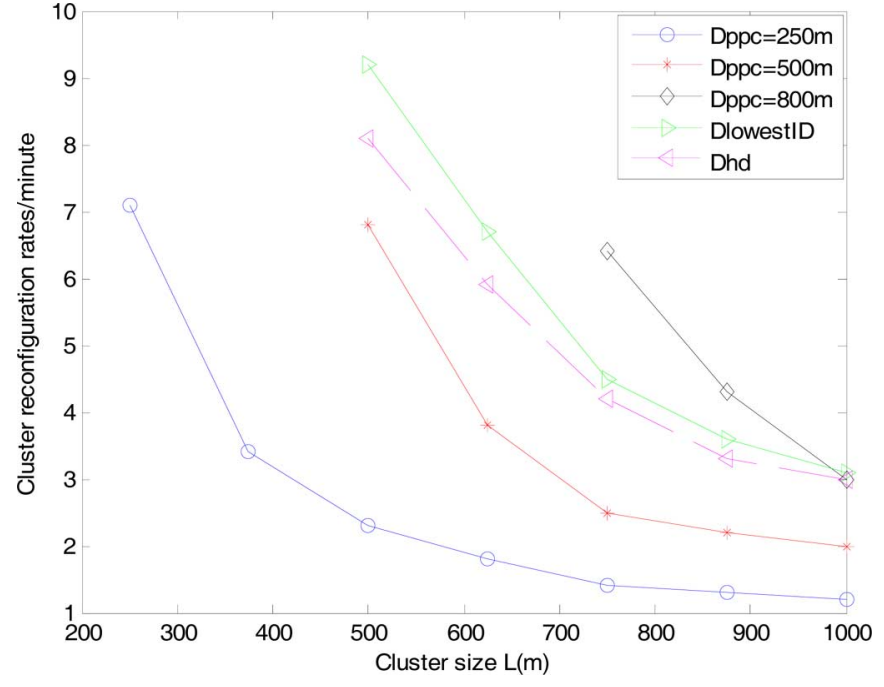

Fig. 9. Cluster reconfiguration rate versus cluster size.

than or equal to the cluster dismiss threshold. As a result, the probability of a cluster being dismissed also increases. Second, increasing the cluster dismiss thresholds decreases the probability that a member of the dismissed cluster successfully finds a neighboring cluster to join.

For a fixed value of the cluster dismiss threshold $D$, the cluster reconfigured rate decreases as the cluster radius $L$ increases. There are two reasons for this. First, when the cluster radius is larger, the members of the dismissed clusters have higher probability of finding neighboring clusters to join, resulting in the lower probability of forming one or more new clusters. Second, when a node moves away from its cluster head such that the distance from its cluster head is greater than $L$, larger cluster radius results in higher probability for the node to find a cluster head within $L$; therefore, the probability of forming a new cluster is lower.

The average aggregated link throughput versus transmission range is shown in Fig. 10. As a comparison, the lowest ID and highest degree clustering methods are also simulated to find the relationship between radio transmission range and the average aggregated link throughput. The results show that the proposed method significantly outperforms the traditional ad hoc clustering techniques in terms of throughput. The proposed method is more dedicated to fast-topology-changing ad hoc networks with high mobility.

The results confirm that there exists a tradeoff between transmission range and throughput. For a relatively smaller transmission range, the graph consists of several isolated subgraphs, with good spatial reuse but poor connectivity. Too small transmission range, however, leads to throughput decrease, since most of the clusters contain only one node and no links. As the transmission range grows, the proposal has better connectivity but less efficient spatial reuse, and thus, lower throughput.

Based on the earlier simulations, it is clearly shown that larger cluster size and smaller dismiss threshold lead to low cluster reconfiguration rate, which is equivalent to higher network stability. The radio transmission range of most wireless ad hoc 


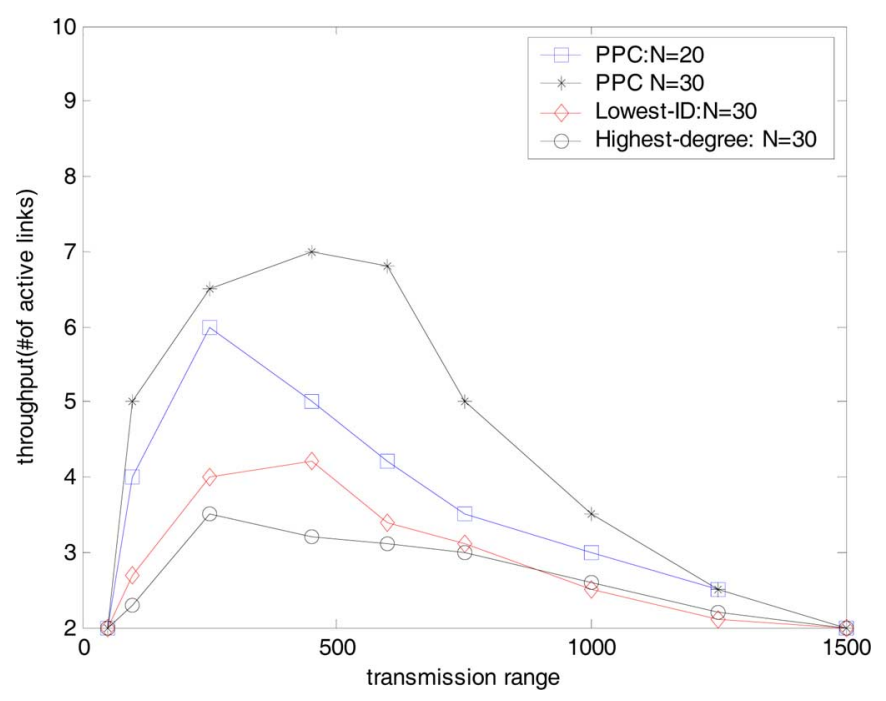

Fig. 10. Link throughput comparisons.

devices varies from 125-1000 m [6], [10], [12], [22]. However, from Fig. 10, we observe that the highest throughput can be achieved if the radius is from $250-700 \mathrm{~m}$. Therefore, the ideal cluster size is about $800-1000 \mathrm{~m}$, and the dismiss threshold is about $200-300 \mathrm{~m}$, while a highway has a two-lane spatial capacity and moderate average velocity.

\section{SUMMARY}

In this paper, a new clustering technique for ad hoc vehicle networks is proposed. To achieve the stable cluster structure, a cluster is controlled by the priorities associated with vehicles' traffic information together with their geographical position information. Unlike regular MANETs, the performance of VANETs highly depends on vehicle traffic status. The predefined maximum distance between the cluster head and its members, then, controls the cluster size. It enables nodes to move during cluster setup and maintenance. The dismiss threshold controls the cluster reconfiguration. VANET can be considered as a 1-D network by taking the number of lanes into account. This paper performs basic mathematical analysis of PPC's performance under some assumptions. Simulations show that this new technique has nice flexibility and stability. Future research should include the influence in terms of available bandwidth and capacity. Studies of extreme cases such as sparse and jammed traffic should also be included in the future work. Security and other performance issues should be considered as well [24]-[28].

\section{REFERENCES}

[1] A. Amis, R. Prakash, T. Vuong, and D. T. Huynh, "MaxMin D-cluster formation in wireless ad hoc networks," in Proc. IEEE Conf. Comput. Commun., Tel Aviv, Israel, Mar. 1999, vol. 1, pp. 32-41.

[2] A. Amis and R. Prakash, "Load-balancing clusters in wireless ad hoc networks," in Proc. 3rd IEEE Symp. Appl.-Specific Syst. Softw. Eng. Technol., Richardson, TX, Mar. 2000, pp. 25-32.

[3] M. Aoki and H. Fuji, "Inter-vehicle communication: Technical issues on vehicle control application," IEEE Commun. Mag., vol. 34, no. 10, pp. 99-93, Oct. 1996.
[4] B. Awerbuch and D. Peleg, "Sparse partitions," in Proc. 31st Annu. Symp. Found. Comput. Sci., 1990, pp. 503-513.

[5] C. Alwan, R. Bagrodia, N. Bambos, M. Gerla, L. Kleinrock, J. Short, and J. Villasenor, "Adaptive mobile multimedia networks," IEEE Pers. Commun., vol. 3, no. 2, pp. 34-51, Apr. 1996.

[6] L. Bao and J. J. Garcia-Luna-Aceves, "Topology management in ad hoc networks," in Proc. 4th ACM Int. Symp. Mobile Ad Hoc Netw. Comput., 2003, pp. 129-140.

[7] D. J. Baker, A. Ephremides, and J. A. Flynn, "The design and simulation of a mobile radio network with distributed control," IEEE J. Sel. Areas Commun., vol. SAC-2, no. 1, pp. 226-237, Jan. 1984.

[8] C. Bettstetter and R. Krausser, "Scenario-based stability analysis of the distributed mobility-adaptive clustering (DMAC) algorithm," in Proc. 2nd ACM Int. Symp. Mobile ad hoc Netw. Comput., Long Beach, CA, 2001, pp. 232-241.

[9] S. Biswas, R. Tatchikou, and F. Dion, "Vehicle-to-vehicle wireless communication protocols for enhancing highway traffic safety," IEEE Commun. Mag., vol. 44, no. 1, pp. 28-29, Jan. 2006.

[10] J. Blum, A. Eskandarian, and L. Hoffman, "Challenges of intervehicles ad hoc networks," IEEE Trans. Intell. Transp. Syst., vol. 5, no. 4, pp. 347351, Dec. 2004.

[11] J. Broch, D. A. Maltz, D. B. Johnson, Y. Hu, and J. Jetcheva, "A performance comparison of multi-hop wireless ad hoc network routing protocols," in Proc. 4th ACM Annu. Int. Conf. Mobile Comput. Netw., Dallas, TX, Oct. 1998, pp. 85-97.

[12] Z. Chen, H. Kung, and D. Vlah, "Ad hoc relay wireless networks over moving vehicles on highways," in Proc. 2nd ACM Int. Symp. Mobile Ad Hoc Netw. Comput., Long Beach, CA, 2001, pp. 247-250.

[13] M. Gerla and J. Tsai, "Multicluster, mobile, multimedia radio network," ACM/Wireless Netw., vol. 1, no. 3, pp. 255-265, 1995.

[14] H. Hartenstein, B. Bochow, and D. Vollmer, "Position-aware ad hoc wireless networks for inter-vehicle communications: The Fleetnet project," in Proc. 2nd ACM Int. Symp. Mobile Ad Hoc Netw. Comput., Long Beach, CA, 2001, pp. 259-262.

[15] T. Kosch and C. Schwingenschloegl, "Mobile ad hoc testbed for vehicleto-vehicle communication," in Proc. 5th Int. Conf. Syst., Cybern. Inf. (SCI) VTC2000, 2001, pp. 1545-1549.

[16] T. Marc, F. Andreas, and H. Hannes, "System design for information dissemination in VANETs," in Proc. 3rd Int. Workshop Intell. Transp. (WIT), 2006, pp. 27-33.

[17] K. Nakao, K. Saita, N. Karasawa, M. Sengoku, and S. Shinoda, "Teletraffic characteristics in cellular systems and traffic flow in road sytems," in Proc. IEEE 49th Veh. Technol. Conf., 1999, vol. 2, pp. 1193-1197.

[18] M. Ravi, C. Adithya, S. Mohan, and M. Ranga, "Reliable MAC broadcast protocol in directional and omni-directional transmissions for vehicular ad hoc networks," in Proc. 2nd ACM Internet Workshop Veh. Ad Hoc Netw. (VANET 2005), Cologne, Germany, pp. 10-19.

[19] H. Reijimers and R. Prasad, "The influence of vehicle distribution models on packet success probability on a three lane motorway," in Proc. IEEE Veh. Technol. Conf.,, 1998, pp. 1785-1789.

[20] H. Takagi and L. Kleinrock, "Optimal transmission ranges for randomly distributed packets radio terminals," IEEE Trans. Commun., vol. 32, no. 3 , pp. 246-257, Mar. 1984.

[21] A. T. Toyserkani, E. Strom, and A. Svensson, "An efficient broadcast MAC scheme for traffic safety applications in automotive networks," in Proc. Wireless Commun. Netw. Conf., Las Vegas, NV, 2006, pp. 21002105.

[22] B. Xu, A. Ouksel, and O. Wolfson, "Opportunistic resource exchange in inter-vehicle ad-hoc networks," IEEE Int. Conf. Mobile Data Manage., Jan. 2004, pp. 45-51.

[23] U. S. DOT: The National ITS Architecture Version 3.0, 1999.

[24] M. Yu, K. Leung, and A. Malvankar, "A dynamic clustering and energy efficient routing technique for sensor networks," IEEE Trans. WirelessCommun., vol. 6, no. 8, pp. 3069-3079, Aug. 2007.

[25] M. Yu, A. Malvankar, and W. Su, "An environment monitoring system architecture based on sensor networks," Int.J.Intell. Control Syst., vol. 10, no. 3, pp. 201-209, Sep. 2005.

[26] Z. Jiang and M. C. Zhou, "Spread spectrum MAC protocol with dynamic rate and collision avoidance for mobile ad hoc network," IEEE Trans. Veh. Technol., vol. 52, no. 5, pp. 3149-3158, Sep. 2007.

[27] C. Zhang and M. C. Zhou, "A stochastic Petri net approach to modeling and analysis of ad hoc networks," Int. J. Intell. Control Syst., vol. 8, no. 1 , pp. 8-19, Jan. 2003.

[28] C. Zhang, M. C. Zhou, and M. Yu, "Ad hoc network routing and security: A review," Int. J. Commun. Syst., vol. 20, pp. 909-925, Aug. 2007. 


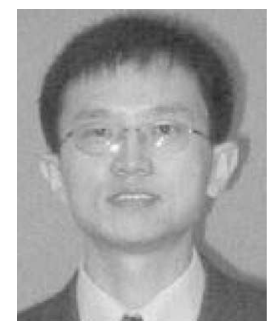

Zhigang Wang (S'01-M'06) received the B.S. and M.S. degrees in electrical engineering from the University of Electronic Science and Technology, Chengdu, China, in 1995 and 1999, respectively, and the $\mathrm{Ph} . \mathrm{D}$. degree in electrical engineering from the New Jersey Institute of Technology, Newark, in 2006.

$\mathrm{He}$ is currently an Assistant Research Professor at the Center for Advanced Vehicular Systems, Mississippi State University, Starkville. His current research interests include wireless ad hoc networking technologies in intelligent transportation systems, signal processing in sensor networks, and mesh networks.

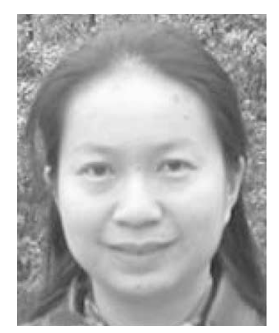

Lichuan Liu (S'01-M'06) received the B.S. and M.S. degrees in electrical engineering from the University of Electronic Science and Technology, Chengdu, China, in 1995 and 1998 respectively, and the Ph.D. degree in electrical engineering from the New Jersey Institute of Technology, Newark, in 2006.

She is currently an Assistant Professor at the Department of Electrical Engineering, Northern Illinois University, Dekalb. Her current research interests includes space-time signal coding, crosslayer design in wireless sensor networking, ad hoc networking, and digital signal processing.

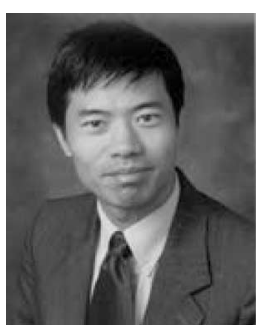

MengChu Zhou (S'88-M'90-SM'93-F'03) received the B.S. degree in electrical engineering from Nanjing University of Science and Technology, Nanjing, China in 1983, M.S. degree in automatic control from Beijing Institute of Technology, Beijing, China, in 1986, and the Ph.D. degree in computer and systems engineering from Rensselaer Polytechnic Institute, Troy, NY, in 1990.

He joined the New Jersey Institute of Technology (NJIT), Newark, in 1990, where he is currently a Professor of electrical and computer engineering and the Director of the Discrete Event Systems Laboratory. He is the author or coauthor of about 300 publications including six books, more than 120 journal papers, and 16 book chapters. He is a coauthor with F. DiCesare, Petri Net Synthesis for Discrete Event Control of Manufacturing Systems (Kluwer Academic, 1993), editor of Petri Nets in Flexible and Agile Automation (Kluwer Academic, 1995), coauthor with K. Venkatesh, Modeling, Simulation, and Control of Flexible Manufacturing Systems: A Petri Net Approach (World Scientific, 1998), coeditor with M. P. Fanti, Deadlock Resolution in Computer Integrated Systems (Marcel Dekker, 2005), coauthor with H. Zhu, Object-Oriented Programming in C++: A Project-Based Approach (Tsinghua University Press, 2005), and coauthor with B. Hruz, Modeling and Control of Discrete Event Dynamic Systems (Springer, 2007). He is an Editor-in-Chief of the International Journal of Intelligent Control and Systems. His current research interests include Petri nets, computer-integrated systems, wireless ad hoc and sensor networks, system security, semiconductor manufacturing, and embedded control.
Prof. Zhou was invited to lecture in Australia, Canada, China, France, Germany, Hong Kong, Italy, Japan, Korea, Mexico, Taiwan, and United States, and served as a plenary speaker for several conferences. He was an Associate Editor of the IEEE TRANSACTIONS ON ROBOTICS AND AUTOMATION from 1997 to 2000, and the IEEE TRANSACTIONS ON AUTOMATION SCIENCE AND ENGINEERING from 2003 to 2007, and is currently the Managing Editor of the IEEE TRANSACTIONS ON SYSTEMS, MAN, AND CYBERNETICS: PART C, an Associate Editor of the IEEE TRANSACTIONS ON SYSTEMS, MAN, AND CYBERNETICS: PART A and the IEEE TRANSACTIONS ON INDUSTRIAL INFORMATICS. He served as a Guest Editor for many journals including the IEEE TRANSACTIONS ON INDUSTRIAL ELECTRONICS and the IEEE TRANSACTIONS ON SEMICONDUCTOR MANUFACTURING. He was the General Co-Chair of the 2003 IEEE International Conference on System, Man, and Cybernetics (SMC), Washington DC, October 5-8, the Founding General Co-Chair of the 2004 IEEE International Conference on Networking, Sensing and Control, Taipei, Taiwan, R.O.C, March 21-23, and the General Chair of the 2006 IEEE International Conference on Networking, Sensing and Control, Ft. Lauderdale, FL, April 23-25. He was the Program Chair of the 1998 and 2001 IEEE International Conference on SMC and the 1997 IEEE International Conference on Emerging Technologies and Factory Automation. He also served other chair positions including Local Arrangement Chair of the 2007 American Control Conference, New York, July 2007. He is serving as the General Chair of the IEEE Conference on Automation Science and Engineering, Washington, DC, August 23-26, 2008. He organized and chaired over 80 technical sessions and served on program committees for many conferences. He has led or participated in over 30 research and education projects with total budget over $\$ 10$ million, funded by the National Science Foundation (NSF), Department of Defense, Engineering Foundation, New Jersey Science and Technology Commission, and industry. He was the recipient of the NSF's Research Initiation Award, the Computer Integrated Manufacturing (CIM) University Leadership and Excellence in the Application and Development of Integrated Manufacturing (LEAD) Award by the Society of Manufacturing Engineers, the Perlis Research Award by NJIT, the Humboldt Research Award for the U.S. Senior Scientists, the Leadership Award and Academic Achievement Award by the Chinese Association for Science and Technology - USA, the Asian American Achievement Award by the Asian American Heritage Council of New Jersey, and the Distinguished Lecturer of the IEEE SMC Society. He was the Founding Chair of the Discrete Event Systems Technical Committee of the IEEE SMC Society, and the Chair of the Semiconductor Manufacturing Automation Technical Committee of the IEEE Robotics and Automation Society. He is a Life Member of the Chinese Association for Science and Technology_USA and was its President in 1999.

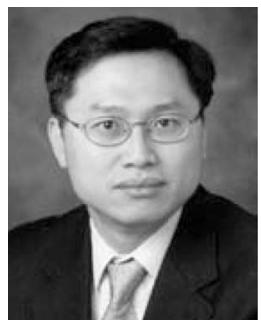

Nirwan Ansari (S'78-M'83-SM'94) received the B.S. degree (summa cum laude) in electrical engineering from the New Jersey Institute of Technology (NJIT), Newark, in 1982, the M.S. degree in electrical engineering from the University of Michigan, Ann Arbor, in 1983, and the Ph.D. degree in electrical engineering from Purdue University, West Lafayette, IN, in 1988 .

Since 1988, he has been with the Department of Electrical and Computer Engineering, NJIT, where he is currently a Professor and the Director of Advanced Networking Lab. He is the author or coauthor of more than 300 papers and the coauthor of Computational Intelligence for Optimization (Springer, 1997) and Neural Networks in Telecommunications (Springer, 1994). His current research interests include broadband networks and multimedia communications.

Dr. Ansari is a Senior Technical Editor of the IEEE COMMUNICATIONS MAGAZINE, and is on the editorial board of Computer Communications, the ETRI Journal, and the Journal of Computing and Information Technology. He was the Founding General Chair of the First IEEE International Conference on Information Technology: Research and Education (ITRE, 2003) and the Chair of the IEEE North Jersey Section and a member of the IEEE Region 1 Board of Governors during 2001-2002. He has been involved in various IEEE activities such as the Chair of Communications Society (COMSOC) Technical Committee on Ad Hoc and Sensor Networks, and Chair/Vice-Chair of several conferences/symposia. His is the recipient of the NJIT Excellence Teaching Award in Graduate Instruction in 1998, the IEEE Region 1 Award in 1999, and an IEEE Leadership Award in 2007. He was also an IEEE Communications Society Distinguished Lecturer. 